\title{
Underwater locomotion from oscillatory shape deformations
}

\author{
Patricio A. Vela ${ }^{\dagger}$, Kristi A. Morgansen ${ }^{\dagger}$ and Joel W. Burdick ${ }^{\ddagger}$ \\ ${ }^{\dagger}$ Control and Dynamical Systems $\quad \ddagger$ Mechanical Engineering \\ California Institute of Technology, Mail Code 107-81, Pasadena, CA, 91125 \\ \{pvela,kristi\}@cds.caltech.edu, jwberobotics.caltech.edu
}

\begin{abstract}
This paper considers underwater propulsion that is generated by variations in body shape. We summarize and extend some of the emerging approaches for the uniform modeling and control of such underactuated systems. Two examples illustrate these ideas.
\end{abstract}

\section{Introduction}

This paper considers the self-propulsion of deformable bodies in (primarily ideal) fluids. We are principally motivated by an interest in robotic underwater vehicles that propel and steer themselves by changes in shape (e.g. $[11,13,15]$ and the references therein), rather than by conventional propellers and maneuvering surfaces. The purpose of this paper is to suggest that a common framework for analyzing and controlling these systems is emerging. On the mechanics side, the use of symmetry and reduction principles leads to governing equations that have useful geometric structure $[1,11,13]$. On the control side, we introduce averaging-based control methods that are suited to the stabilization of such underactuated systems by periodic feedback.

The biologically-inspired systems we consider here produce propulsive forces through the use of oscillatory shape change, and one appropriate literature for motion generation in similar systems comes from the use of feedforward sinusoidal control inputs for kinematic and dynamic underactuated control systems $[3,6,8,17]$. Extending these feedforward results to appropriate feedback inputs that produce stabilization and tracking in the general setting is nontrivial and the construction often difficult, but results are available for particular driftless system structures $[2,4,16,20]$. Recently, the use of averaging has produced oscillatory feedback results with simpler construction that apply to classes of configuration controllable dynamic systems $[4,21]$. Here we show the extension of the relevant averaging and feedback results to driftless control systems. We illustrate these ideas with two examples: an amoeba-like system and a simplified planar model of a carangiform fish.

\section{Underwater Mechanical Systems}

A deformable swimmer's configuration can be decomposed into two types of coordinates. The "group" or "position" coordinates, denoted by $g$, describe the location of a body-fixed

\footnotetext{
${ }^{1}$ This work was supported in part by an NSF Engineering Research Center grant (NSF9402726) and NSF grant CMS-9502224.
}

reference frame relative to a fixed reference frame. These coordinates form the Lie group $S E(3)$, or one of its subgroups. The "shape" variables, denoted by $r$, describe the vehicle's internal configuration. Together these variables form a principal bundle. The vehicle's position and orientation are indirectly controlled: shape variations couple through physical constraints to generate motion in the group variables.

\subsection{Lagrangian Mechanics}

In the most general form, the mechanics of a deformable swimmer can be quite involved. As the body deforms, the fluid is perturbed. The perturbed fuid in turn influences the body's motion. Thus, the general problem involves a set of coupled dynamics on an infinite dimensional phase space. We adopt a Lagrangian viewpoint for these systems. Neglecting potential energy terms for the moment, the Lagrangian consists of the kinetic energy of the body and the fluid:

$$
\mathcal{L}=\frac{1}{2} \dot{q}^{T} \mathcal{M}(q) \dot{q}+\frac{\rho}{2} \int_{\mathcal{D}} m\left(\dot{\chi}_{t}, \dot{\chi}_{t}\right) \mu
$$

where $q=\left[g^{T}, r^{T}\right]^{T}$ is the vehicle configuration, $\chi_{t}$ denotes a fluid particle position at time $t, \mathcal{M}$ and $m$ are the rigid body and fluid kinetic energy metrics, and $\mu$ is the volume element. The fluid of density $\rho$ fills a domain $\mathcal{D}$ outside the body. The system may also be subject to constraints (e.g. the Kutta condition on lifting surfaces).

While the general problem of fluid modeling for control is beyond the scope of this paper, we note that progress can be made by using inherent symmetries and by making simplifying assumptions on the nature of the fluid flow to reduce the system to a finite dimensional state space. The two examples in this paper are made tractable by this line of reasoning. We first consider an amoeba-like model, whose surrounding flow can be well-approximated by potential flow. The second example, which models a real planar robot fish prototype, achieves simplicity because the influence of shed vorticity on the body's movement is ignored. In these examples and others, the fluid flow can be reduced to a function of the body's velocity. We henceforth assume this simplification in our control developments.

Essentiaily all deformable swimmers fit within the class of general mechanical systems, whose equations of motion as derived from the Lagrangian take the form

$$
M(q) \ddot{q}+C(q, \dot{q}) \dot{q}+B(q)=E(q, \dot{q})+\hat{X}_{a}(q) v^{a}(t)
$$


where summation over upper and lower indices is assumed, $q \in \mathbb{R}^{n}, u \in \mathbb{R}^{m}, M$ is the mass matrix (or kinetic energy metric), $C$ contains Coriolis and centrifugal tcrms, $B$ contains potential forces such as gravity, $E$ are applied forces on the system (such as drag), and $\hat{X}$ are the control vector fields. Typically $E$ decomposes into state-feedback terms $E_{0}(q, \dot{q})$, dissipative forces $-E_{1}(q) \dot{q}$, and external forcing $E_{2}(q, \dot{q})$. Solving for acceleration gives

$$
\ddot{q}=S(q, \dot{q})+X_{0}(q, \dot{q})-D(q) \dot{q}+X_{a}(q) v^{a}(t)
$$

where the drift term is $S(q, \dot{q})=-M^{-1}(q)(C(q, \dot{q})+$ $\left.E_{(q, \dot{q})}+B(q)\right)$, and the drag term is $D(q) \dot{q}=$ $M^{-1}(q) E_{1}(q) \dot{q}$. The time-varying vector fields are $X_{a}(q)=$ $M^{-1}(q) \hat{X}_{a}(q)$, while the time-invariant state feedback controls are contained in $X_{0}(q, \dot{q})=M^{-1}(q) E_{0}(q, \dot{q})$.

The above system (1) can be converted to first order representation. Letting $x=\left[q^{T}, \dot{q}^{T}\right]^{T}$, the equations become

$$
\dot{x}=S(x)+Y_{0}(x)-D(x)+Y_{a}(x) v^{a}(t)
$$

with $x(0)=x_{0}$. Systems of this type have many favorable mathematical properties, including Lie-algebraic ones [19]. For example, the Jacobi-Lie brackets between input vector fields of a mechanical system vanish:

$$
\left[Y_{a}, Y_{b}\right]=0
$$

The Jacobi identity implies that $\left[Y_{a},\left[S, Y_{b}\right]\right]=\left[Y_{b},\left[S, Y_{a}\right]\right]$. Hence, one may define a symmetric product

$$
\left\langle Y_{a}: Y_{b}\right\rangle=\left[Y_{b},\left[S, Y_{a}\right]\right]
$$

A second important equality is the following:

$$
\left[Y_{c},\left[Y_{b},\left[Y_{a}, S+Y_{0}-D\right]\right]\right]=0,
$$

which occurs when the polynomial dependence of the vectors fields $S, Y_{0}$, and $D$ do not exceed order 2 in the velocities, which is true for most mechanical systems.

The principal bundle structure of the configuration space alluded to earlier can be used to obtain further refinements. Almost universally, the Lagrangian and the inherent mechanical constraints of deformable propulsors are invariant with respect to the Lie group $S E(3)$ or one of its subgroups. Consequently, one can apply reduction theory $[1,9]$ to simplify the resulting equations of motion and to expose useful geometric structure in the mechanics. In the absence of constraints, the equations of motion are given by Hamel's equations [1]:

$$
\frac{d}{d t} \frac{\partial \mathcal{L}}{\partial \xi}=a d_{\xi}^{*} \frac{\partial \mathcal{L}}{\partial \xi}+F_{\xi}, \quad \frac{d}{d t} \frac{\partial \mathcal{L}}{\partial \dot{r}}=\frac{\partial \mathcal{L}}{\partial r}+F_{r},
$$

where $\xi=g^{-1} \dot{g}$ is the vehicle's velocity (in body coordinates), $F_{\xi}=F_{\xi}(g, r, \xi, \dot{r})$ and $F_{r}=F_{r}(r, \dot{r}, t)$ are forces acting on the mechanism in the position and shape directions, and $\mathcal{L}$ is the system Lagrangian. Additional flow assumptions can lead to further simplifications. For example, in the presence of momentum preservation constraints, reduction principles lead to reduced equations of the form [11]:

$$
\begin{aligned}
g^{-1} \dot{g} & =-A(r) \dot{r}+\left(\Lambda^{g g}\right)^{-1}(r) p \\
\dot{p} & =\operatorname{ad}_{\xi}^{*} p \\
\mathcal{M}(r) \ddot{r} & =T(r) \tau-B(r, \dot{r})
\end{aligned}
$$

where $p$ is a bulk system momentum (in body coordinates), and $\tau$ denotes the "shape forces." With respect to the amoeba-like example, this structure arises from the overall conservation of body/fluid momentum due to the $S E(2)$ invariance of the Lagrangian. The first equation is termed the reconstruction equation, with $A$ being the local connection and $\Lambda^{g g}$ the locked inertia tensor. The second equation describes the momentum evolution (in body coordinates). In spatial coordinates, this momentum is constant. In the case of zero initial momentum, the momentum evolution is trivial. The third equation describes the "shape" dynamics. Assuming that we have complete control over the shape variables, in the zero momentum case, the system equations can be put into the form of a driftless affine control system (10) with $q=\left[g^{T}, r^{T}\right]^{T}$ the state and $\dot{r}$ the control inputs

$$
\left[\begin{array}{c}
\dot{g} \\
\dot{r}
\end{array}\right]=\left[\begin{array}{c}
-g A(r) \\
I
\end{array}\right] \dot{r} \Rightarrow \dot{q}=Y_{a}(q) v^{a}
$$

This equation shows the importance of the local connection to these problems. In the more general case when the fluid motion is not a function only of the vehicle's motion, generalizations of the concepts reviewed here apply.

\section{Representative Systems}

We consider two deformable underwater propulsors. The first example, an amoeba-like locomotor, represents systems dominated by added-mass effects, while the second example, a carangiform-like robot fish, represents systems with strong inertial and lifting effects. This section briefly describes appropriate system models. More details can be found in the references.

\subsection{An Amoeba-like Propulsor}

Real amoebae are microscopically small. They operate at a very low Reynolds number, and the relevant fluid equations are those of creeping flow ${ }^{1}$. However, the Reynolds number of a macroscopic "robot amoeba" operating in water would be much higher. We thus make the reasonable idealization that the amoeba is a connected deformable body swimming through an inviscid and incompressible fluid. We also assume that the fluid is irrotational, and that the amoeba cannot generate vorticity-i.e., the amoeba does not have sharp fins. The position of the body's center of mass and the amoeba's orientation are denoted by $g \in S E(2)$ relative to a bodyfixed frame. Any "amoeba" robot which we might actually construct would have a finite number of actuators. Hence, we assume the body's boundary can be described by a finite number of shape variables $r$.

\footnotetext{
'Surprisingly, creeping flow can also be put in this framework.
} 


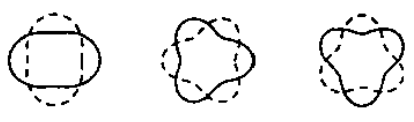

Figure 1: Amoeba-like system with three deformation modes.

The kinetic energy of the amoeba-fluid system consists of both the swimmer's and the fluid's kinetic energies. Using the group invariance of the Lagrangian, and letting $\phi$ denote the fluid potential, one can show the following.

Proposition 1 ([11]) The G-invariant kinetic energy of a deformable body in a potential flow has the reduced form

$$
T_{\text {total }}(r, \dot{r}, \xi)=\frac{1}{2}\left[\xi^{T}, \dot{r}^{T}\right]\left[\begin{array}{cc}
I & I A \\
A^{T} I & m
\end{array}\right]\left[\begin{array}{c}
\xi \\
\dot{r}
\end{array}\right]
$$

where $I \in \mathbb{R}^{6 \times 6}$ is the "locked added inertia" matrix with

$$
(I)_{i j}=\Lambda_{i j}^{g g}(r)-\frac{\rho_{0}}{2} \int_{\Sigma}\left(\phi_{i}^{g}\left(\nabla \phi_{j}^{g} \cdot n\right)+\phi_{j}^{g}\left(\nabla \phi_{i}^{g} \cdot n\right)\right) d S
$$

where $\rho_{0}$ is the fluid density, $\xi=g^{-1} \dot{g}$, and the potential takes the linear form: $\sum_{i} \phi_{i}^{g} \xi_{i}+\sum_{j} \phi_{j}^{s} \dot{r}_{j}$. The term $\Lambda^{g g}(s)$ is the vehicle's "locked inertia matrix" (considered in the absence of the fluid), and the second term is the classical added fluid mass/inertia. The matrix $I A \in \mathbb{R}^{6 \times n_{r}}$ has entries

$$
(I A)_{i j}=\Lambda_{i j}^{g s}(s)-\frac{\rho_{0}}{2} \int_{\Sigma}\left(\phi_{i}^{g}\left(\nabla \phi_{j}^{s} \cdot n\right)+\phi_{j}^{s}\left(\nabla \phi_{i}^{g} \cdot n\right)\right) d S .
$$

The local connection form, $A$, can be computed as

$$
A(s)=I^{-1}(s)(I A)(s)
$$

As an example, assume that the boundary of a circular amoeba-like vehicle with nominal radius $r_{0}$ can deform according to the three "modes" seen in Figure 1. The amoeba's radius, $R$, at each boundary point is

$$
R(\sigma, r)=r_{0}\left[1+\epsilon\left(r_{1} \cos (2 \sigma)+r_{2} \cos (3 \sigma)+r_{3} \sin (3 \sigma)\right)\right]
$$

where $\epsilon$ is a small parameter prescribing the deformation's magnitude, and $r_{1}(t), r_{2}(t), r_{3}(t)$ represent the three deformation modes. The $1^{\text {st }}$ and $2^{\text {nd }}$ modes together yield motion in the $x$-direction, the $1^{s t}$ and $3^{\text {rd }}$ modes together yield motion in the $y$-direction, and the $2^{\text {nd }}$ and $3^{\text {rd }}$ modes together yield motion in the $\theta$-direction. Based on formulas (8) and (9), one can show [11] that the local connection form for this example is

$A=\epsilon^{2}\left[\begin{array}{ccc}r_{0}(1-\alpha) r_{2} & r_{0} r_{1} & 0 \\ r_{0}(1-\alpha) r_{3} & 0 & r_{0} r_{1} \\ 0 & \frac{-2 \pi r_{0}^{2} \rho r_{3}}{M} & \frac{2 \pi r_{0}^{2} \rho r_{2}}{M}\end{array}\right]+\mathcal{O}\left(\epsilon^{3}\right)$

where $\alpha=\left(2 \pi r_{0}^{2} \rho\right) /\left(M+\pi r_{0}^{2} \rho\right)$ and $g^{-1} \dot{g}=[\dot{x}, \dot{y}, \dot{\theta}]^{T}$.

\subsection{Carangiform Locomotion}

The simple fish-like robot discussed in this paper and related earlier works $[5,10,12,14]$ consists of a planar three-link

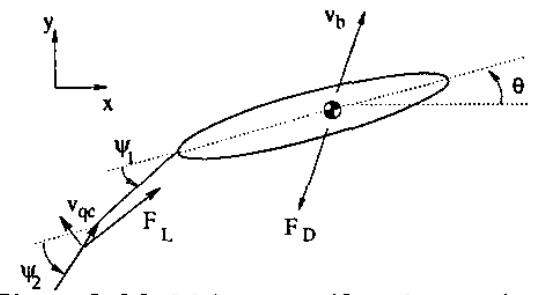

Figure 2: Model for carangiform locomotion.

mechanism immersed in water (see Fig. 2). The orientation of the peduncle and tail joints are denoted by $r=\left[\psi_{1}, \psi_{2}\right]^{T}$, and are measured with respect to the main body reference frame. The forces acting on the system are lift on the tail and drag on the body. The comparatively small effects of lift on the body, form drag on the tail, skin friction, and shed vorticity are ignored. As discussed in [14], the drag for a translating and rotating plate is taken to be

$$
F_{D}=\frac{1}{2} \rho C_{d} h \int_{\frac{1}{2}-a}^{\frac{l}{2}+a}\left\|\xi\left((a+s) e_{1}\right) \times e_{1}\right\| \xi\left((a+s) e_{1}\right) d s
$$

and the associated moment to be

$M_{D}=\frac{1}{2} \rho C_{d} h \int_{\frac{l}{2}-a}^{\frac{1}{2}+a}\left\|\xi\left((a+s) e_{1}\right) \times e_{1}\right\|\left(\xi\left((a+s) e_{1}\right) \times e_{1}\right) s d s$

where $\rho$ is the fluid density, $l$ is the plate length, $C_{d}$ is the plate's drag coefficient when its velocity lies in the $y$ direction, $h$ is the plate height, $a$ is the difference in position between the plate's center of mass and center of geometry, and $\xi\left((a+s) e_{1}\right)$ is an infinitesimal generator giving the bodyfixed velocity of the plate at the point $a+s$ along the body. The value of $s$ varies from $\frac{l}{2}-a$ to $\frac{l}{2}+a$, and the unit vector $e_{1}$ is in the direction of the body-fixed $x$ axis. The lift acting on a flat plate is

$$
F_{L}=\pi \rho A\left(\xi_{q c} \times e_{t}\right) \times \xi_{q c}
$$

where $\xi_{q c}$ is the velocity at the plate's quarter chord point as measured in the body frame, $e_{t}$ is a unit vector pointing along the plate toward its leading edge and $A$ is the plate's area. These equations are a simplification via reduction of those from [14], in recognition of the position invariant nature of the lift and drag forces.

\section{Averaging}

Oscillatory actuation is a natural approach for achieving full control of shape-controlled underactuated mechanical systems. Averaging theory, a useful tool for simplifying complex systems undergoing periodic forcing, converts the nonautonomous vector fields into autonomous vector fields. The retained time-averaged terms illuminate the contribution of the oscillatory controls. These averaging coefficients are denoted by

$$
\mathrm{V}_{(n)}^{(a)}(t)=\int_{0}^{t} \int_{0}^{s_{n-1}} \cdots \int_{0}^{s_{2}} v^{a}\left(s_{1}\right) d s_{1} \cdots d s_{n-1} .
$$

For the case where there are multiple upper and lower indices, assume that they are the product of the above type of integral. 
As an example

$$
\mathrm{V}_{(1,1)}^{(a, b)}(t)=\mathrm{V}_{(1)}^{(a)} \mathrm{V}_{(1)}^{(b)}=\left(\int_{0}^{t} v^{a}\left(s_{1}\right) d s_{1}\right)\left(\int_{0}^{t} v^{b}\left(s_{1}\right) d s_{1}\right)
$$

The time average of an averaging coefficient results in an averaged coefficient, $\overline{\mathrm{V}}(t)=\frac{1}{T} \int_{0}^{T} \mathrm{~V}(t) d t$. Additionally define $\tilde{V}_{(n)}^{(a)}=V_{(n)}^{(a)}-\bar{V}_{(n)}^{(a)}$ and for the multi-index version $\tilde{\mathrm{V}}_{(N)}^{(A)}=\mathrm{V}_{(N)}^{(A)}-\overline{\mathrm{V}_{(N)}^{(A)}}$ where $(A)=\left(a_{1}, a_{2}, \ldots, a_{|A|}\right)$ and $(N)=\left(n_{1}, n_{2}, \ldots, n_{|N|}\right)$.

Control systems are usually classed into two categories: with or without drift. Prior work on averaging for underactuated mechanical systems has primarily focused on those systems for which accessibility and controllability can be achieved with first order Lie brackets or symmetric products. As discussed in [21,22], however, a number of interesting systems require higher order effects in order to achieve a full range of motions. The carangiform fish robot is a particular example of such a-system as it can realize a turning maneuver using second level symmetric products. Previously ([21]) the authors developed a second-order âveraging technique for such systems with drift. Similar results hold for systems without drift. Below we first present a new theorem for driftless systems, followed by the analogous results for those with drift.

Consider the driftless affine control system,

$$
\dot{q}=Y_{a}(q) u^{a}(q, t),
$$

defined on some domain $D \subset \mathrm{R}^{n}$. For oscillatory inputs $u^{a}(q, t)=f^{a}(q)+v^{a}(t / \epsilon)$,

$$
\dot{q}=Y_{a}(q) f^{a}(q)+Y_{a}(q) v^{a}(t / \epsilon)
$$

where $v^{a}(t)$ are $T$-periodic functions with zero average, and $f^{a}(q)$ is a state dependent term that can be used to stabilize the directly controlled states. A transformation of time puts it into the required averaging form,

$$
\frac{\mathrm{d} q}{\mathrm{~d} \tau}=\epsilon Y_{a}(q) f^{a}(q)+\epsilon Y_{a}(q) v^{a}(t) .
$$

Theorem 2 Consider the system (11) and the initial value problem

$$
\begin{aligned}
\dot{z}= & Y_{a}(z) f^{a}(z)+\epsilon \overline{\mathrm{V}_{(1)}^{(a)}(t)}\left[Y_{a}(z), Y_{b}(z) f^{b}(z)\right] \\
& +\epsilon \overline{\mathrm{V}_{(1,0)}^{(a, b)}(t)}\left[Y_{a}(z), Y_{b}(z)\right]
\end{aligned}
$$

with $z(0)=q_{0}$. If the control vector fields and the inputs are Lipschitz continuous in the state, continuous in both state and time, and the solution $z(t) \in D$, then $q(t)-z(t)=O(\epsilon)$ as $\epsilon \rightarrow 0$ on the time scale 1. Furthermore, $q(t)-z(t)=O(\epsilon)$ as $\epsilon \rightarrow 0$ for all time if $z=0$ is an asymptotically stable critical point of the linear approximation of (13).

Proof: See appendix.
Systems with drift have an analogous structure with added terms representing dynamic effects. A standard form for oscillatory control of systems with drift is

$$
\dot{x}=S(x)+Y_{0}(x)-D(x)+\frac{1}{\epsilon} Y_{a}(x) v^{a}(t / \epsilon)
$$

where $x=(q, \dot{q}), S(x)$ is the drift, $Y_{0}(x)$ denotes statefeedback terms, and $D(x)$ denotes dissipative terms.

Theorem 3 (Second order averaging [21]) Consider the system (14) and the initial value problem

$$
\begin{aligned}
& \dot{z}=S(z)+Y_{0}(z)-D(z)-\mathrm{V}_{(1,1)}^{(a, b)}\left\langle Y_{a}: Y_{b}\right\rangle \\
& +\frac{1}{2} \epsilon \overline{\mathrm{V}_{(2,1)}^{(a, b)}(t)}\left[\left[Y_{a}, S+Y_{0}-D\right],\left[Y_{b}, S+Y_{0}-D\right]\right] \\
& \left.-\frac{1}{2} \epsilon \overline{\left(\mathrm{V}_{\langle 2,1,1)}^{(a, b, c)}(t)\right.}-\overline{\mathrm{V}_{(2)}^{(a)}} \overline{\mathrm{V}_{(1,1)}^{(b, c)}}\right) \cdot\left\langle Y_{a}:\left\langle Y_{b}: Y_{c}\right\rangle\right\rangle
\end{aligned}
$$

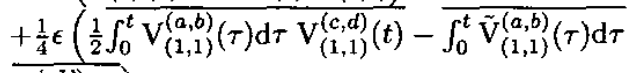

$$
\begin{aligned}
& \left.\overline{\mathrm{V}_{(1,1)}^{(c ;)}(t)}\right) \cdot\left[\left\langle Y_{a}: Y_{b}\right\rangle,\left\langle Y_{c}: Y_{d}\right\rangle\right]
\end{aligned}
$$

with $z(0)=z_{0}$. If the control vector fields and input forcing are smooth functions of their respective arguments, and the Lie bracket properties of (3) and (5) hold, then $x(t)-\Phi_{t}^{g}(z(t))=O(\epsilon)$ as $\epsilon \rightarrow 0$ on the time scale 1 . Furthermore $x(t)-\Phi_{t}^{g}(z(t))=O(\delta(\epsilon))$ as $\epsilon \rightarrow 0$ for all. $t$, if $z=0$ is an asymptotically stable critical point for the linear approximation of the system in (15).

\section{Motion Generation}

In order to track trajectories, we must be able to generate motion in arbitrary directions in state space. In the two classes of systems which we are considering, these directions are characterized by Lie brackets for kinematic systems and symmetric products for configuration controllable dynamic systems (for a discussion of configuration controllability, see [7]).

Motion generation and open loop trajectory tracking have been studied extensively for driftless nonholonomic systems $[8,15,17,20]$. However, while it is straightforward to generate motion along one Lie bracket direction, trajectory tracking requires simultaneously and independently generating motion along all directions necessary for controllability. As we show in this section and the next, this task can be accomplished through appropriate use of feedback.

In systems of the form (10), $m$ states are directly controlled, and Lie brackets are formed from the vector field $Y_{a}$. As is known from the above references, generating motion along a direction corresponding to a Lie bracket $\mathcal{B}$ of order $p$ can then be accomplished with inputs of the form $\phi_{I}=\alpha \cos (p \omega t), \phi_{I I}=\sin (\omega t)$. At least one vector fields $Y_{a}$ will occur only once in $\mathcal{B}$. We then assign $\phi_{I}$ to the control input $u_{a}$ and $\phi_{I I}$ to the controls that correspond to the remainder of the vector fields occurring in $\mathcal{B}$. To independently generate motion in all directions simultaneously, we 
superimpose the controls for each direction and use a different frequency band for each motion. We will assign frequencies starting with first level Lie bracket motions and work up through increasing levels. Start with $\omega_{1}=1$. Let $p_{i}$ be the order of the next Lie bracket to have an assigned frequency and $p_{i-1}$ the order of the Lie bracket for the previously assigned frequency. Then we choose the new frequency

$$
\omega_{i}= \begin{cases}p_{i} \omega_{i-1}+1, & \text { if } p_{i}=p_{i-1} \\ p_{i-1} \omega_{i-1}+1, & \text { if } p_{i}>p_{i-1}\end{cases}
$$

and subscript the corresponding $\alpha$ as $\alpha_{i}$. This choice of controls will move the system independently in any direction, and the amount of motion in the different directions can be scaled with the use of $\alpha$. Superimposing the contributions from each bracket to be generated results in controls of the form

$$
u^{a}(t)=\sum_{i} \alpha_{i} \cos \left(p_{i} \omega_{i} t\right)+\sum_{j} \sin \left(\omega_{j} t\right)
$$

Matters are a bit more difficult for dynamic systems; but a corresponding result holds. Specifically we are interested in systems which are configuration controllable with symmetric products up to second level. If the system (14) is configuration controllable of order 2, we know that there exists a set of linearly independent vector fields $Y_{a},\left\langle Y_{a}: Y_{b}\right\rangle$, $\left\langle Y_{a}:\left\langle Y_{b}: Y_{c}\right\rangle\right\rangle$ that span $\mathbb{R}^{n}$. For the elements $Y_{a b}=$ $\left\langle Y_{a}: Y_{b}\right\rangle$ from this set define

$$
\zeta_{a b}^{a}=\alpha_{a b} \cos \left(\lambda_{a b} t\right), \zeta_{a b}^{b}=\cos \left(\lambda_{a b} t\right),
$$

where $\lambda_{a b} \in \mathbb{Z}^{+}$, the $\alpha_{a b}$ are scalar constants and the $\lambda_{a b}$ are chosen as in (16) with $p=1$. For the elements $Y_{a b c}=$ $\left\langle Y_{a}:\left\langle Y_{b}: Y_{c}\right\rangle\right\rangle$ define

$$
\xi_{a b c}^{I}=\alpha_{a b c} \cos \left(\nu^{I} \lambda_{a b c} t\right), \xi_{a b c}^{I I}=\cos \left(\nu^{I I} \lambda_{a b c} t\right)
$$

where $\lambda_{a b c} \in \mathbb{Z}^{+}$and the $\alpha_{a b c}$ are scalar constants. Unless $a=b=c$, at least one index in $\left\langle Y_{a}:\left\langle Y_{b}: Y_{c}\right\rangle\right\rangle$ occurs uniquely. To the control corresponding to this unique index assign $\xi_{a b c}^{I}$, and to the other two assign $\xi_{a b c}^{I I}$. If an index is repeated, choose $\nu^{I}=2$ and $\nu^{I I}=1$. If no index is repeated, choose $\nu^{I}=3$, and assign $\xi_{a b c}^{I}$ to $u^{a}$. To $u^{b}$ assign $\xi_{a b c}^{I I}$ with $\nu^{I I}=2$, and to $u^{c}$ assign $\xi_{a b c}^{I I}$ with $\nu^{I I}=1$. The frequencies $\lambda_{a b c}$ are chosen according to

$$
\lambda_{112}=3 \lambda_{m-1, m}, \lambda_{i j k}=\lambda_{\{i j k\}-1}+3 \lambda_{m-1, m}+1 .
$$

Now sum the appropriate components to get

$$
u^{a}(t)=\sum_{i j} \zeta_{i j}^{a}+\sum_{i j k} \xi_{i j k}^{a}
$$

\section{Trajectory Stabilization}

Given the previous averaging and motion generation results, we can now apply state feedback to stabilize underwater system response to a desired trajectory.

For driftless systems controllable with first level Lie brackets, such as the amoeba, the system average was given above.
Motivated by this result and those in $[8,17]$, we can assume that any kinematic system controllable with Lie brackets up to level $p$ with inputs (17) can be expressed as

$$
\dot{z}=Y_{a}(z) f^{a}(z)+\sum_{i=1}^{p} \mathcal{L}^{i}(z) H_{i}(\alpha)=\mathcal{L}(z) H(\alpha)
$$

where $\mathcal{L}^{i}(z)$ is a matrix whose columns are all the Lie brackets of level $i$ and $H_{i}(\alpha)$ are the corresponding parameterized coefficients from the previous section.

Theorem 4 Consider a kinematic system of the form (10), which is controllable with Lie brackets up to level $p$ and where the dimension of the subspace spanned by the brackets at level $i$ is $n_{i}$. Assume that there exist functions of the form (17) such that the linearization of $H(\alpha)$ with respect to $\alpha$ at $\alpha=0$ is invertible on the subspace to control, and let $z(t)$ be the averaged system response. Then there exists $K \in \mathbb{R}^{\left(\sum n_{i}\right) \times n}$ such that for

$$
\alpha=-\Lambda K z(T\lfloor t / T\rfloor)
$$

where $\alpha \in \mathbb{R}^{\sum n_{i}}$ and $\Lambda \in \mathbb{R}^{(n-m) \times(n-m)}$, we have the stabilized average system response $\lim _{t \rightarrow \infty} z(t)=0$.

Proof: See appendix.

Using the averaged system response for a second order system with the parameterized controls (20), we can rewrite the averaged dynamics as

$$
\dot{z}=S(z)+Y_{0}(z)-D(z)+B(z) H(\alpha)
$$

Theorem 5 ([21]) Consider a mechanical system of the form (14), which is configuration controllable with first and second level symmetric products, and where the dimensions of the spaces spanned by $Y_{a},\left\langle Y_{a}: Y_{b}\right\rangle$, and $\left\langle Y_{a}:\left\langle Y_{b}: Y_{c}\right\rangle\right\rangle$ are respectively, $m, n_{a b}$, and $n_{a b c}$. Assume that there exist functions of the form (18) and (19) such that the linearization of $H(\alpha)$ with respect to $\alpha$ at $\alpha=0$ is invertible on the subspace to control, and let $z(t)$ be the averaged system re sponse. Then there exists $K \in \mathbb{R}^{\left(n_{a b}+n_{a b c}\right) \times 2 n}$ such that for

$$
\alpha=-\Lambda K z(T\lfloor t / T\rfloor)
$$

where $\alpha \in \mathbb{R}^{n_{a b}+n_{a b c}}$ and $\Lambda \in \mathbb{R}^{(n-m) \times(n-m)}$ is an invertible matrix, we have the stabilized average system response $\lim _{t \rightarrow \infty} z(t)=0$.

\section{Simulation Results}

To demonstrate these ideas, we will consider the two example systems introduced above. As discussed, the amoeba is a driftless, kinematic system and the fish is an underactuated mechanical system. Using the three mode shapes discussed in Sec. 3, the amoeba is free to move in the plane 


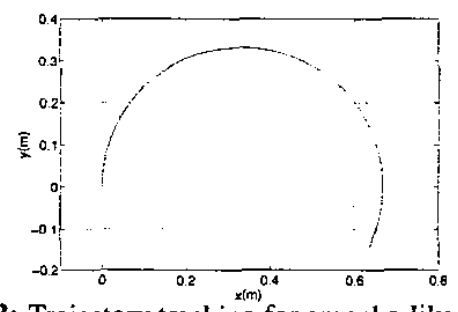

Figure 3: Trajectory tracking for amoeba-like system.

and is thus controllable. Simulation results for a desired helical trajectory are shown in Fig. 3 for $M=0.01 \mathrm{~kg}$ and $r_{0}=0.2 \mathrm{~m}$. The orientation remained constant, while the error was at most on the order of $3 \%$ of body radii. The shape went through about 1000 iterations and traveled a total distance on the order of 6 body radii, which is consistent with the calculation in [11].

As derived, the carangiform fish is described by Hamel's equations (6) and more generally by (2). A symmetric product can be used to generate forward locomotion, and the Lie bracket between the drift and the peduncle angle control vector fields can be used to generate rotation while in motion. While this effect comes from a first level Lie bracket, a slower turn can also be effected through a second level symmetric product. Study of such effects requires the higher order averaging and motion generation treated above. Results for such effects can be found in $[14,15]$. The first order symmetric product and Lie bracket for this system evaluated at the reference configuration $\left(\left[\psi_{1}, \psi_{2}\right]=[0,0]\right)$ for typical physical parameters (see [15]) give, for the group variables,

$$
\begin{aligned}
\left\langle Y_{1}: Y_{2}\right\rangle & \approx(0.15,0,0)^{T} \\
{\left[Y_{1}, S+Y_{0}-D\right] } & \approx\left(f\left(\xi_{2}, \xi_{3}\right), 0.27 \xi_{1},-18.70 \xi_{1}\right)^{T}
\end{aligned}
$$

where $f$ is a function of the lateral and rotational body velocities, $\xi_{2}$ and $\xi_{3}$, respectively. The symmetric product demonstrates that forward locomotion is theoretically possible. The Lie bracket shows that a turning motion is achievable; it must be mentioned that the lateral translation and the turning do not oppose each other in directing the carangiform fish to reduce the error. Thus, in the plane we have rudimentary control over forward motion and bearing, which can be used for locomotion. The results of tracking a straight line along the $x$-axis given an initial position error are shown in Fig. 4. Steady state error is within $5 \%$ and could be eliminated by including an integral term in the feedback control functions (23). Actual experimental results can be found in [15].
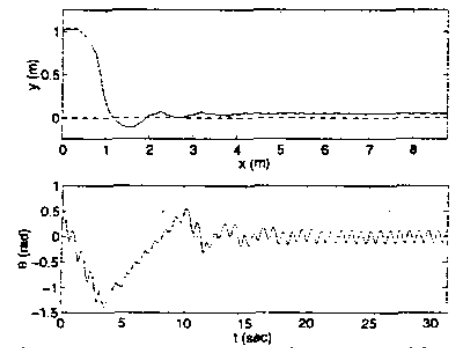

Figure 4: Trajectory tracking for carangiform fish.

\section{Conclusion}

The mechanics of deformable underwater propulsors operating in simple fluids satisfy several properties that hold in most operating regimes. Furthermore, we have found that oscillatory controls and averaging techniques apply quite generally to such systems. Their Lie-algebraic structure simplifies the averaging process for systems with drift and their typical $S E(3)$ invariance allows for reduction.

Two examples, an amoeba-like swimmer and a carangiformlike mechanism, illustrated these ideas. By using simplifying fluid dynamic principles, both models were rendered finitedimensional, and therefore tractable. The amoeba can successfully track nearly arbitrary planar trajectories, while the carangiform system can track straight and curved trajectories.

Ongoing research seeks to more deeply understand the universal nature of such systems at a more fundamental level, specifically with the inclusion of fluid dynamics in the principal bundle structure. The averaging process to arbitrary order will also be critical for more complicated systems requiring higher order brackets.

\section{References}

[1] A.M. Bloch, P.S. Krishnaprasad, J.E. Marsden, and R.M. Murray. Nonholonomic mechanical systems with symmetry. Arch. Rational Mech. Anal., 126(1):21-99, 1996.

[2] A.M. Bloch, M. Reyhanoglu, and N.H. McClamroch. Control and stabilization of nonholonomic dynamic systems. IEEE Trans. Aut. Contr., 37(11):1746-57, Nov. 1992.

[3] F. Bullo. Averaging and vibrational control of mechanical systems. SIAM J. Contr. Optim., 2001. submitted.

[4] F. Bullo, N.E. Leonard, and A.D. Lewis. Controllability and motion algorithms for underactuated Lagrangian systems on Lie groups. IEEE Trans. Aut. Contr., 45(8):1437-54, 2000.

[5] S.D. Kelly, R.J. Mason, C.T. Anhalt, R.M. Murray, and J.W. Burdick. Modelling and experimental investigation of carangiform locomotion for control. In Proc. Amer. Contr. Conf., pages 1271-6, 1998.

[6] N.E. Leonard. Periodic forcing, dynamics and control of underactuated spacecraft and underwater vehicles. In Proc. 34th IEEE Conf. Dec. Cont., pages 1131-6, 1995.

[7] A.D. Lewis and R.M. Murray. Configuration controllability of simple mechanical control systems. SIAM J. Contr. Optim., 35(3):766-90, 1997.

[8] W. Liu. An approximation algorithm for nonholonomic systerns. SIAM J. Contr. Optim., 35(4):1328-65, 1997.

[9] J.E. Marsden and T.S. Ratiu. Introduction to Mechanics and Symmetry. Springer-Verlag, New York, 1994.

[10] R.J. Mason and J.W. Burdick. Construction and modelling of a carangiform robotic fish. In Proc. 1999 Int. Symp. Exp. Rob., pages 235-42, 1999.

[11] R.J. Mason and J.W. Burdick. Propulsion and control of deformable bodies in an ideal fluid.In Proc. IEEE Int. Conf. Rob. Aut., 1999.

[12] R.J. Mason and J.W. Burdick. Experiments in carangiform robotic fish locomotion. In Proc. IEEE Int. Conf. Rob. Aut., pages $428-35,2000$. 
[13] K.A. McIsaac and J.P. Ostrowski. Motion planning for dynamic eel-like robots. In Proc. Int. Conf. Rob. Aut., pages 1695-700, 2000.

[14] K.A. Morgansen, V. Duindam, R.J. Mason, J.W. Burdick, and R.M. Murray. Nonlinear control methods for planar carangiform robot fish locomotion. In Proc. IEEE Int. Conf. Rob. Aut., pages $427-34,2001$.

[15] K.A. Morgansen, P.A. Vela, and J.W. Burdick. Trajectory stabilization for a planar carangiform robot fish. In Proc. IEEE Int. Conf. Rob. Aut., pages 756-762, 2002.

[16] P. Morin, J.-B. Pomet, and C. Samson. Design of homogeneous time-varying stabilizing control laws for driftless controllable systems via oscillatory approximation of Lie brackets in closed loop. SIAM J. Contr. Optim., 38(1):22-49, Dec. 1999.

[17] R.M. Murray and S. Sastry. Nonholonomic motion planning: Steering using sinusoids. IEEE Trans. Aut. Contr., 38(5):700-16, May 1993.

[18] J.A. Sanders and F. Verhulst. Averaging Methods in Nonlinear Dynamical Systems. Springer-Verlag, 1985.

[19] E.D. Sontag and H.J. Sussman. Time-optimal control of manipulators. Proc. of IEEE lnt. Conf. on Robotics and Automation, pages 1646-1652, 1986.

[20] A.R. Teel, R.M. Murray, and G. Walsh. Nonholonomic control systems: From steering to stabilization with sinusoids. In Proc. 31st IEEE Conf. Dec. Contr, pages 1603-9, 1992.

[21] P. A. Vela, K. A. Morgansen, and J. W. Burdick. Second order averaging methods for oscillatory control of underactuated mechanical systmes. In Proc. Amer. Contr. Conf., pages 4672-7, 2002.

[22] P.A. Vela, K.A. Morgansen, and J.W. Burdick. Second order averaging methods and oscillatory feedback control of underactuated mechanical systems. SIAM J. Contr. Optim., 2002. Submitted.

\section{A Proofs}

Proof of Theorem 2: We will use the theorem of Sanders and Verhulst [18] as it applies to affine control systems. Transform time to the form in (12). For the average, we will need to calculate

$$
\dot{z}=\epsilon \bar{F}(z)+\epsilon^{2} \bar{G}(z)
$$

The first order average is easily seen to be

$$
\tilde{F}(z)=Y_{a}(z) f^{a}(z)
$$

The second order term begins with

$$
G(z, t)=\frac{1}{2} \cdot\left[\int_{0}^{t} Y_{a}(z) v^{a}(z, \tau) \mathrm{d} \tau, Y_{b}(z) v^{b}(z, t)\right] .
$$

Using linearity of the Lie bracket,

$$
\begin{aligned}
G(z, t)= & \frac{1}{2}\left[\int_{0}^{t} Y_{a}(z) f^{a}(z) \mathrm{d} \tau, Y_{b}(z) f^{b}(z)\right] \\
& +\frac{1}{2}\left[\int_{0}^{t} Y_{a}(z) u^{a}(t) \mathrm{d} \tau, Y_{b}(z) f^{b}(z)\right] \\
& +\frac{1}{2}\left[\int_{0}^{t} Y_{a}(z) f^{a}(z) \mathrm{d} \tau, Y_{b}(z) u^{b}(t)\right] \\
& +\frac{1}{2}\left[\int_{0}^{t} Y_{a}(z) u^{a}(t) \mathrm{d} \tau, Y_{b}(z) u^{b}(t)\right]
\end{aligned}
$$

From the time independence of the vector fields, and the skew-symmetry of the Lie bracket, the first term vanishes.
For the average we get

$$
\begin{aligned}
\bar{G}(z)= & \frac{1}{2 T} \int_{0}^{T}\left[\int_{0}^{t} Y_{a}(z) u^{a}(t) \mathrm{d} \tau, Y_{b}(z) f^{b}(z)\right] \mathrm{d} t \\
& +\frac{1}{2 T} \int_{0}^{T}\left[\int_{0}^{t} Y_{a}(z) f^{a}(z) \mathrm{d} \tau, Y_{b}(z) u^{b}(t)\right] \mathrm{d} t \\
& +\frac{1}{2 T} \int_{0}^{T}\left[\int_{0}^{t} Y_{a}(z) u^{a}(t) \mathrm{d} \tau, Y_{b}(z) u^{b}(t)\right] \mathrm{d} t
\end{aligned}
$$

Using integration of products and the zero-average assumption of the time dependent inputs, the two mixed terms can be consolidated. Moving the time dependent coefficients outside the Lie brackets,

$\bar{G}(z)=\overline{\mathrm{V}_{(1)}^{(a)}(t)}\left[Y_{a}(z), Y_{b}(z) f^{b}(z)\right]+\frac{1}{2} \overline{V_{(1,0)}^{(a, b)}(t)}\left[Y_{a}(z), Y_{b}(z)\right]$

All together, the averaged system is

$$
\begin{aligned}
\dot{z}= & \epsilon Y_{a}(z) f^{a}(z)-\epsilon^{2} \overline{\mathrm{V}_{(1)}^{(a)}(t)}\left[Y_{a}(z), Y_{b}(z) f^{b}(z)\right] \\
& +\frac{1}{2} \epsilon^{2} \overline{\mathrm{V}_{(1,0)}^{(a, b)}(t)}\left[Y_{a}(z), Y_{b}(z)\right],
\end{aligned}
$$

which after a transformation of time, and application of the relevant theorems in [18] gives the desired results.

Proof of Theorem 4: Given the assumptions on the system, the averaged system (21) is controllable. Linearizing the system with respect to $z$ and $\alpha$ yields

$$
\dot{z}=\left.\frac{\partial(\mathcal{L} H(\alpha))}{\partial z}\right|_{z, \alpha=0} z+B(0) \frac{\partial H}{\partial \alpha} \alpha=A z+\left[\begin{array}{c}
0 \\
\tilde{B} \Gamma
\end{array}\right] \alpha .
$$

For the averaging result to hold, the system parameters $\alpha$ must be constant over whole periods. Allowing the values of the parameters to be modified at the endpoints of each whole period results in a discrete time system, the dynamics are obtained by direct integration of (25):

$$
z(T)=e^{A T} z(0)+e^{A T} \int_{0}^{T} e^{-A \tau} d \tau\left[\begin{array}{c}
0 \\
\tilde{B} \Gamma
\end{array}\right] \alpha .
$$

To perform this computation, note that the matrix $A$ can always be block diagonalized by a state space transformation such that the real part of the eigenvalues of the upper left block (of dimension $m \times m$ ) correspond to the states that are directly controlled and in the average are all negative, those of the middle block are either positive or negative and those of the lower right block are all zero. We will assume that $A$ in (26) is in this block diagonal structure. Now we effectively have the discrete, linear system

$$
z(h+1)=\hat{A} z(h)+\hat{B} \alpha(h)
$$

where $\hat{A}=\operatorname{diag}\left[e^{A_{1} T}, e^{A_{2} T}, I\right]$ and $\hat{B}=\left[\begin{array}{cc}0 & 0 \\ M \tilde{B} \Gamma & \end{array}\right]$ with

$$
M=\left[\begin{array}{cc}
e^{A_{2} T} A_{2}^{-1}\left(I-e^{-A_{2} T}\right) & 0 \\
0 & T I+\frac{1}{2} T^{2} J_{0}
\end{array}\right]
$$

where $J_{0}$ is a matrix with ones on the superdiagonal and zeros elsewhere. The proof is completed by finding any matrix $K$ such that with $\alpha(h)=-(M \tilde{B} \Gamma)^{-1} K z(h)=-\Lambda K z(h)$, the eigenvalues of $\hat{A}-\hat{B} K \Lambda$ are within the unit circle. 